\title{
Candida tropicalis as an emerging pathogen in Candida meningitis: case report and review
}

\begin{abstract}
Candida species are an uncommon cause of meninigitis. Given the rarity of this infection, the epidemiology, prognosis, and optimal therapy for Candida meningitis are poorly defined. The authors report on a paraplegic patient due to spinal cord injury who developed C. tropicalis meningitis. In addition, we review and discuss other reported cases of C. tropicalis meningitis in the medical literature.
\end{abstract}

Keywords: Candida; meningitis.

[Braz J Infect Dis 2010;14(6):631-633]@Elsevier Editora Ltda.

\section{INTRODUCTION}

Candida species are an uncommon cause of meningitis. Given the rarity of this infection, the epidemiology, prognosis, and optimal therapy of Candida meningitis are poorly defined. Risk factors for meningitis are similar to those associated with invasive candidiasis. The risk of developing this complication is unknown. It occurs in immunosupressed patients, in patients treated with broad-spectrum antibiotics and receiving parenteral nutrition or result of disseminated disease. ${ }^{1-4}$ In addition, two specific patient groups, premature neonates and neurosurgical patients, are at increased risk.

The case of a paraplegic patient due to a spinal cord injury who developed C. tropicalis meningitis is herein reported and the cases reported in the medical literature are reviewed and discussed medical literature.

\section{CASE REPORT}

A 26-year-old man was admitted to Hospital das Clínicas, Porto Alegre, Brazil, in February 2009 complaining of nausea, vomiting, headache and fever $\left(39^{\circ} \mathrm{C}\right)$. Physical exam revealed neck stiffness without any focal neurological signs. Previous medical history included paraplegia due to a spinal injury (2002), decubitus ulcers (2002), pelvic osteomyelitis (2006) and recurrent urinary tract infections. A lumbar puncture yielded cerebrospinal fluid (CSF) with 2,500 leukocytes $/ \mathrm{mm}^{3}$ (100\% neutrophils), an elevated protein level of $98 \mathrm{mg} / \mathrm{dL}$, and a reduced glucose level of $34 \mathrm{mg} / \mathrm{dL}$. No fungi and bacteria were seen on Gram stain. CSF cultures were negative for bacteria and fungi. Chest-X ray, head CT scan, and transesophageal echocardiogram results were normal. Blood cultures and HIV serologic test results were negative. Urine cultures grew a mixed flora of Gram-positives and negatives, but urinary Gram-stain revealed innumerous yeasts compatible with Candida spp. Despite broad-spectrum 96-hours antibiotic therapy including cefepime, vancomycin, and metronidazole, the patient persisted with fever and headache. Repeated lumbar puncture showed 106 leukocytes $/ \mathrm{mm}^{3}$ (70\% neutrophils, 30\% lymphocytes), a protein level of $40 \mathrm{mg} / \mathrm{dL}$, and a glucose level of $24 \mathrm{mg} / \mathrm{dL}$. At 24 hours, the primary plates and broth culture grew a budding yeast that was identified with a $99 \%$ probability as C. tropicalis on API 20C (bioMérieux). The isolates fluconazole MIC was $0.025 \mu \mathrm{g} / \mathrm{mL}$ on disk diffusion susceptibility testing. The patient was treated with amphotericin $\mathrm{B}$ deoxycholate $(1.0 \mathrm{mg} / \mathrm{kg} / \mathrm{day})$ for five days but progressed to respiratory insufficiency, coma and death.
Authors

Luciano Z Goldani ${ }^{1}$ Rodrigo P Santos ${ }^{2}$

${ }^{1}$ Infectious Diseases Unit, Hospital de Clínicas de Porto Alegre, Universidade Federal do Rio Grande do Sul, Brazil.

${ }^{2}$ Infectious Diseases Unit, Hospital de Clínicas de Porto Alegre, Universidad Federal do Rio Grande do Sul, Brazil.

Submitted on: 03/05/2010 Approved on: 05/21/2010

Correspondence to: Dr. Luciano Z. Goldani Infectious Diseases Unit Hospital de Clínicas de Porto Alegre

Ramiro Barcelos 2350 Porto Alegre, 900640-003 Brazil

We declare no conflict of interest. 


\section{DISCUSSION}

C. albicans accounts for $70 \%-100 \%$ of all fungal meningitis isolates. ${ }^{3,4}$ Other reported species include $C$. glabrata, C. tropicalis, C. parapsilopsis, and C. lusitaniae. As shown in Table 1, there have been few cas- es of C. tropicalis meningitis described in the medical literature..$^{4-17}$ In contrast to meningitis caused by C. albicans, C. tropicalis meningitis has been increasingly reported in adults. Most cases of $C$. tropicalis are postoperative complications of head and neck surgery, including

Table 1. Reported cases and series of $\mathrm{C}$. tropicalis meningitis

\begin{tabular}{|c|c|c|c|c|c|c|}
\hline $\begin{array}{l}\text { Cases/ } \\
\text { Series }\end{array}$ & Age & Sex & Characteristics & Treatment & Outcome & Reference \\
\hline 1 & 54 & Male & $\begin{array}{l}\text { Mastoid exploration; otitis } \\
\text { chronic }\end{array}$ & AmB1 & Alive & Chattopadhyay 5 \\
\hline 2 & 49 & Male & $\begin{array}{l}\text { Occipital craniotomy; } \\
\text { cerebellar hemorrhage }\end{array}$ & FCZ & Alive & Dawson et al. ${ }^{6}$ \\
\hline 3 & $\begin{array}{c}\text { Full- } \\
\text { term } \\
\text { newborn }\end{array}$ & Male & $\begin{array}{l}\text { Intracranial hemorrhage; } \\
\text { respiratory distress; prolonged } \\
\text { antibiotic therapy }\end{array}$ & $\mathrm{AmB}+5^{\prime}$ Flu2 & Alive & Ahuja et al. ${ }^{7}$ \\
\hline 4 & 54 & Male & $\begin{array}{l}\text { Mastoid exploration, } \\
\text { chronic otitis }\end{array}$ & AmB $+5^{\prime}$ Flu & Alive & Chalwick et al. ${ }^{8}$ \\
\hline 7 & 43 & Male & $\begin{array}{l}\text { Hodgkin's lymphoma; } \\
\text { chemotherapy }\end{array}$ & $\mathrm{AmB}$ & Death & Hernig et al. ${ }^{9}$ \\
\hline 8 & 2 & Female & $\begin{array}{l}\text { Myelomeningocele } \\
\text { correction; ventricular- } \\
\text { peritoneal shunt }\end{array}$ & $\mathrm{AmB}+\mathrm{FCZ3}$ & Alive & Byers et al. ${ }^{10}$ \\
\hline 9 & 66 & Female & Corticosteroids & - & Death & Gorell et al. ${ }^{11}$ \\
\hline 10 & 63 & Male & Syringomyelia & $\mathrm{AmB}+5^{\prime}$ Flu & Alive & $\begin{array}{l}\text { Phanthumchinda } \\
\text { et al. }{ }^{12}\end{array}$ \\
\hline 11 & 51 & Female & $\begin{array}{c}\text { Neurinoma, } \\
\text { ventriculo-peritoneal } \\
\text { shunt, hydrocephalia }\end{array}$ & $\mathrm{AmB}+5^{\prime}$ Flu & Alive & Miñambres et al. ${ }^{13}$ \\
\hline 12 & - & - & $\begin{array}{l}7 \text { children with widespread, } \\
\text { leukemia neutropenia, total parental } \\
\text { nutrition,costicosteoids, broad } \\
\text { sprectrum antibiotics }\end{array}$ & Yes & Death & Flynn et al. ${ }^{14}$ \\
\hline 13 & - & - & $\begin{array}{l}11 \text { children with leukemia, } \\
\text { TPN, neutropenia }\end{array}$ & Yes & Death & McCullers et al..$^{15}$ \\
\hline 14 & Newborn & Male & $\begin{array}{c}\text { Sepsis, broad spectrum } \\
\text { antibiotics }\end{array}$ & Yes & Death & Aldress K et al. ${ }^{16}$ \\
\hline 15 & - & - & $\begin{array}{l}\text { Ventriculo-peritoneal shunt, } \\
\text { bacterial meningitis }\end{array}$ & Shunt removal & Alive & $\mathrm{O}^{\prime}$ Brien et al. ${ }^{17}$ \\
\hline 16 & 68 & Female & $\begin{array}{l}\text { Cavernous sinus tumor; } \\
\text { lumbar drainage, bacterial } \\
\text { meningitis, broad spectrum } \\
\text { antibiotic therapy }\end{array}$ & $\begin{array}{c}\mathrm{AMB}+5 \mathrm{FC} \\
\text { shunt removal }\end{array}$ & Alive & Nguyen et al. ${ }^{4}$ \\
\hline 17 & 1 & Female & $\begin{array}{l}\text { Prematurity, hydrocephalus, } \\
\text { peritoneal-ventricular shunt } \\
\text { malfunction }\end{array}$ & $\begin{array}{c}\mathrm{AMB}+5 \mathrm{FC} \\
\text { shunt removal }\end{array}$ & Alive & Chiou et al..$^{18}$ \\
\hline
\end{tabular}

AMB, amphotericin B deoxycholate; 5’Flu, 5’Fluorocytosine; FCZ, fluconazole. 
mastoid exploration, craniotomy, and ventricular-peritoneal shunt. Additional cases of C. tropicalis meningitis in adults have been reported in immunosupressed patients, patients taking prolonged broad-spectrum antibiotic therapy or as a result of disseminated disease, as observed in our case, which developed a possible Candida urinary tract infection with later dissemination to the central nervous system.

Symptoms such as those presented by our patient are similar to other Candida meningitis and include fever, headache, altered mental status, and meningism. Focal neurologic signs are rare. The diagnosis of meningitis is established by a positive CSF culture. Multiple CSF specimens may be required. CSF parameters are variable, with a mild lymphocytic or polymorphonuclear pleocytosis and an increased protein level. Fungal elements are generally not seen. Thus, CSF abnormalities are indistinguishable from cryptococcal, tuberculous, and some bacterial meningitides. Although fluconazole resistant isolates of C. tropicalis have been occasionally reported, the isolate of our case was fluconazole-susceptible.

Despite appropriate therapy with amphotericin B plus 5 fluorocytocine, mortality was seen in 5 of 17 patients (30\%) with C. tropicalis meningitis. In addition to head and neck postoperative procedures, physicians should have a high index of suspicion for Candida meningitis in patients taking broad-spectrum antibiotics who also present an initial source of Candida infection. Non-albicans species identification and appropriate susceptibility tests should be considered for appropriate management of Candida meningitis.

\section{REFERENCES}

1. Fernandez M, Moylett EH, Noyola DE, Baker CJ. Candida meningitis in neonates: a 10-year review. Clin Infect Dis. 2000; 31:458-63.

2. Casado JL, Quereda C, Oliva J, Navas E, Moreno A, Pintado V et al. Candidal meningitis in HIV-infected patients: analysis of 14 cases. Clin Infect Dis. 1997; 25:673-6.

3. Geers TA, Gordon SM. Clinical significance of Candida species isolated from cerebrospinal fluid following neurosurgery. Clin Infect Dis $199 ; 28: 1139-46$.

4. Nguyen MH, Yu V. Meningitis caused by Candida species: an emerging problem in neurosurgical patients. Clin Infec Dis 1995; 21:323-7.

5. Chattopadhyay B. Candida tropicalis meningitis: a case report The Journal of Laryngology \& Otology 1981; 95:1149-51.

6. Dawson NL, Robles HA, Alvarez S. Recurrent Candida tropicalis meningitis. Clinical Neurology and Neurosurgery 2005; 107:243-5.

7. Ahuja SR, Karande S, Kulkarni MV, Tendolkar U. Candida tropicalis meningitis in a young infant. Indian J Pediat 2003; 70:925-7.

8. Chadwick DW, Hartley E, Mackinnon M. Candida tropicalis meningitis Arch Neurol.1980; 37:175-6.

9. Hernig E, Djaldetti M, Pinkhas J, Vries A. Candida tropicalis meningitis in Hodking's disease. JAMA 1967; 119:214-5.

10. Byers M, Chapen S, Feldman A, Parent A. Fluconazole pharmacokinetics in the cerebrospinal fluid of a child with Candida tropicalis meningitis. Pediatr Infect Dis J 1992; 11:895-6.

11. Gorell JM, Palutke WA, Chason JL. Candida pachymeningitis with multiple cranial nerve pareses. Arch Neurology 1979; 36:719-20.

12. Phanthumchinda K, Kaoropthum S. Syringomyelia associated with post-meningitic spinal arachnoiditis due to Candida tropicalis. Postgrad Med J. 1991; 67:767-9.

13. Miñambres E, García-Palomo D, Paternina B, Parra JA, Fariñas MC. Candida tropicalis meningitis associated with external ventricular drainage in an adult female patient. Enfermedades infecciosas y microbiología clínica 2002; 20:94-5.

14. Flynn PM, Marina NM, Rivera GK, Hugher WT. Candida tropicalis infections in children with leukemia. Leukemia and Lymphoma 1993; 10:369-76.

15. McCullers JA et al. Candidal meningitis in children with cancer. Clin Infect Dis, 2000; 31:451-7.

16. Aldress K, Al Shaalan M, Memish Z, Alola S, Bannatyne. Candida meningitis in children: report of two cases. J Chemother 2000; 12:339-44.

17. O'Brien M, Parent A, Davis B. Management of ventricular shunt infections. Childs Brain 1979; 5:304-9.

18. Chiou CC, Wong TT, Lin HH et al. Fungal infections of ventriculoperitoneal shunts in children. Clin Infect Dis 1994; 19:1049-53. 